\title{
Pressure-independent cardiac hypertrophy in mice with cardiomyocyte-restricted inactivation of the atrial natriuretic peptide receptor guanylyl cyclase-A
}

\author{
Rita Holtwick, ${ }^{1}$ Martin van Eickels, ${ }^{2}$ Boris V. Skryabin, ${ }^{3}$ Hideo A. Baba, ${ }^{4}$ \\ Alexander Bubikat, ${ }^{1}$ Frank Begrow,${ }^{1}$ Michael D. Schneider, ${ }^{5}$ David L. Garbers, ${ }^{6}$ \\ and Michaela Kuhn ${ }^{1}$
}

${ }^{1}$ Institut für Pharmakologie und Toxikologie, Universitätsklinikum Münster, Münster, Germany ${ }^{2}$ Physiologisches Institut II, Rheinische Friedrich-Wilhelms-Universität, Bonn, Germany ${ }^{3}$ Institut für Experimentelle Pathologie, Universitätsklinikum Münster, Münster, Germany ${ }^{4}$ Institut für Pathologie, Universitätsklinikum Essen, Essen, Germany

${ }^{5}$ Center for Cardiovascular Development, Baylor College of Medicine, Houston, Texas, USA

${ }^{6}$ Cecil H. and Ida Green Center of Reproductive Biology Sciences, University of Texas Southwestern Medical Center at Dallas, Dallas, Texas, USA

\begin{abstract}
Cardiac hypertrophy is a common and often lethal complication of arterial hypertension. Atrial natriuretic peptide (ANP) has been postulated to exert local antihypertrophic effects in the heart. Thus, a loss of function of the ANP receptor guanylyl cyclase-A (GC-A) might contribute to the increased propensity to cardiac hypertrophy, although a causative role in vivo has not been definitively demonstrated. To test whether local ANP modulates cardiomyocyte growth, we inactivated the GC-A gene selectively in cardiomyocytes by homologous loxP/Cre-mediated recombination. Thereby we have circumvented the systemic, hypertensive phenotype associated with germline inactivation of GC-A. Mice with cardiomyocyte-restricted GC-A deletion exhibited mild cardiac hypertrophy, markedly increased mRNA expression of cardiac hypertrophy markers such as ANP (fivefold), $\alpha$-skeletal actin (1.7-fold), and $\beta$-myosin heavy chain (twofold), and increased systemic circulating ANP levels. Their blood pressure was 7-10 mmHg below normal, probably because of the elevated systemic levels and endocrine actions of ANP. Furthermore, cardiac hypertrophic responses to aortic constriction were enhanced and accompanied by marked deterioration of cardiac function. This phenotype is consistent with a local function of the ANP/GC-A system to moderate the molecular program of cardiac hypertrophy.

J. Clin. Invest. 111:1399-1407 (2003). doi:10.1172/JCI200317061.
\end{abstract}

\section{Introduction}

The heart affects cardiovascular homeostasis by secreting two natriuretic peptides (NPs), atrial natriuretic peptide (ANP) and B-type natriuretic peptide (BNP) (1). They activate a common guanylyl cyclase-A

Received for publication October 3, 2002, and accepted in revised form March 8, 2003.

Address correspondence to: Michaela Kuhn, Institute of Pharmacology and Toxicology, University of Münster, Domagkstrasse 12, D-48149 Münster, Germany. Phone: 49-251-83-52597; Fax: 49-251-83-55501;

E-mail:mkuhn@uni-muenster.de.

Rita Holtwick and Martin van Eickels contributed equally to this work.

Martin van Eickels' present address is: Medizinische UniversitätsPoliklinik, Bonn, Germany.

Conflict of interest: The authors have declared that no conflict of interest exists.

Nonstandard abbreviations used: natriuretic peptide (NP); atrial natriuretic peptide (ANP); B-type natriuretic peptide (BNP); guanylyl cyclase-A (GC-A); natriuretic peptide receptor-C (NPR-C); cardiomyocyte-restricted deletion of GC-A (CM GC-A KO); myosin heavy chain (MHC); periodic acid Schiff (PAS); $\alpha$-skeletal actin $(\alpha$-sk-actin); transverse aortic constriction (TAC); heart weight (HW); body weight (BW); left ventricular weight (LVW).
(GC-A) receptor expressed in a variety of tissues, thereby increasing intracellular cGMP concentrations (2). NPs are secreted from atrial granules into the circulation in response to acute or chronic atrial stretch to act as antihypertensive and antihypervolemic factors through GC-A $(3,4)$. A second receptor subtype that also triggers the effects of NPs is the C-receptor (NP receptor-C, or NPR-C) (4), a clearance receptor that mediates the cellular internalization and degradation of NPs. It may also participate in mediating some of their cellular actions via coupling to $G_{i}$ proteins and inhibition of adenylyl cyclase (5). However, within the cardiovascular system the primary action of the NPR-C seems to be the modulation of circulating and local NP concentrations (6).

In chronic hemodynamic overload, there is a significant increase in ANP and BNP expression in the cardiac ventricles (3). It is postulated that in this situation NPs exert not only endocrine but also local antihypertrophic actions (ANP) and antifibrotic actions (BNP) (7). For example, ANP inhibits growth and proliferation of cultured cardiac myocytes and fibroblasts via GC-A $(8,9)$. Also, overexpression of GC-A in cardio- 

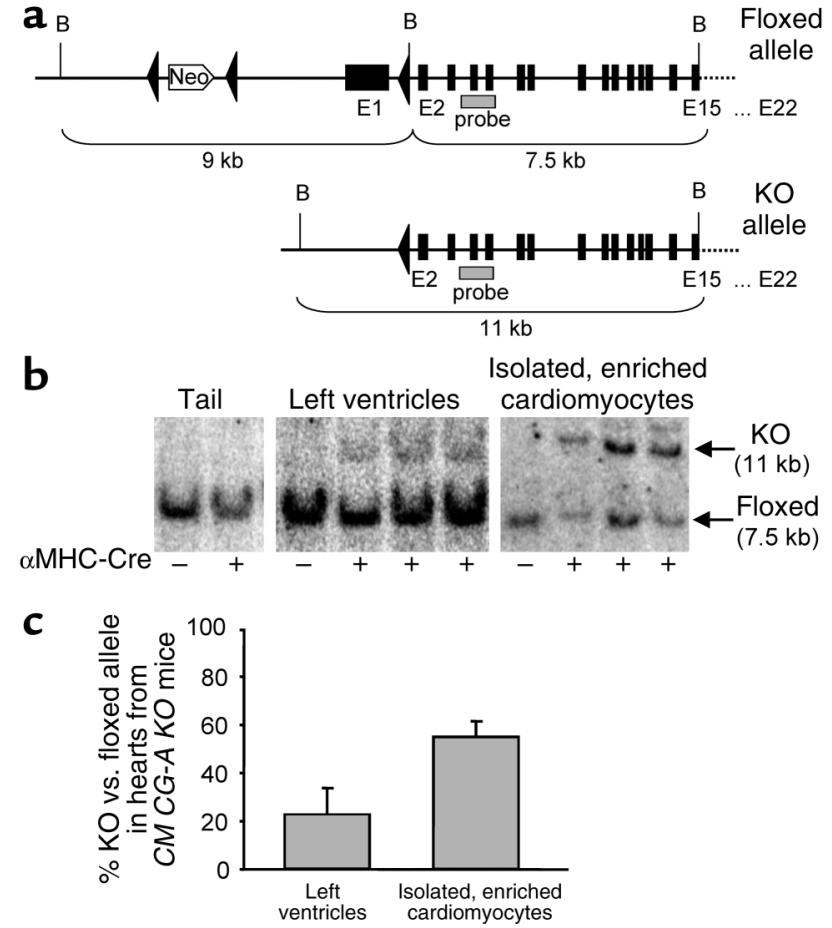

\section{Figure 1}

Generation and Southern blot characterization of "flox/flox GC-A" and "flox/flox GC-A $\times \alpha M H C-C r e^{t g}$ " mice. (a) Restriction map of the targeting construct and the $\alpha \mathrm{MHC}$-Cre-generated deletion allele. $\mathrm{B}$, BamHI site. (b) Southern blot analyses of genomic DNA obtained from tails, left ventricles, and enriched ventricular cardiomyocytes of "flox/flox GC-A" and "flox/flox GC-A $\times \alpha M H C-C r e^{t g}$ " mice, demonstrating the deletion event that occurs in cardiomyocytes from mice harboring the $\alpha M H C-C r e$ transgene (CM GC-A KO mice). The Cremediated deletion event abolishes the additional BamHI site inserted in intron 1 by the recombination event. BamHI-digested DNA was hybridized with a specific cDNA probe (spanning E2-E6); 11-kb and 7.5-kb bands represent the deletion allele and targeted allele, respectively. (c) Efficiency of Cre-mediated GC-A deletion $(n=8)$.

myocytes exerts antihypertrophic effects in vivo (10). Conversely, mice with genetic disruption of the GC-A gene exhibit arterial hypertension and a marked cardiac hypertrophy that is disproportionate to their increased blood pressure and resistant to antihypertensive medication (11-13). By comparison, other mutant mice with similar increases in blood pressure do not have this pronounced hypertrophic phenotype (14). These observations suggest that the ANP/GC-A pathway could be involved in the regulation of myocyte size. However, efforts to prove this concept in vivo have been limited by the systemic, hypertensive phenotype of mice with global deletion of ANP or GC-A $(11,15)$.

To test whether ANP locally modulates cardiomyocyte growth and contractility, we generated mice with selective deletion of GC-A in cardiomyocytes. All endocrine blood pressure- and volume-regulating effects of ANP are preserved. Based on studies in these mice, we conclude that the ANP/GC-A system indeed plays a subtle but critical, autocrine/paracrine role in moderating car- diomyocyte growth and contractile function in vivo independently of its effects on blood pressure.

\section{Methods}

Generation of mice with cardiomyocyte-restricted deletion of GC-A. Cell-selective deletion of GC-A was achieved by the loxP/Cre recombination system. We previously reported the generation of homozygous floxed GC-A mice (16). A neomycin cassette flanked by two loxP sites was placed at $2.6 \mathrm{~kb}$ from exon 1 , upstream from the promoter region of GC-A. A third loxP sequence was placed in intron 1, introducing a new BamHI site for later genotypings. Thereby the promoter region and exon 1 (encoding the signal peptide) were flanked by loxP sites (Figure 1a). As previously demonstrated, expression and function of the floxed GC-A gene are not different from those of the wild-type (16). However, Cre recombinase-mediated deletion is sufficient to inactivate GC-A function completely (16). To achieve a cardiomyocyte-restricted deletion of GC-A, floxed GC-A mice were mated to transgenic mice expressing Cre recombinase under the control of the cardiac $\alpha$-myosin heavy chain promoter $(\alpha M H C$ $\mathrm{Cre}^{\mathrm{tg}}$ mice) (17). The efficiency and specificity of the

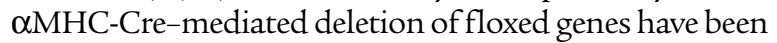
demonstrated by many other studies $(18,19)$. The recombination event in the heart of the resulting "flox/flox $G C-A \times \alpha M H C-C r e^{t g}$ " mice $(C M G C-A K O)$ was detected by Southern blot analysis of genomic DNA isolated from whole hearts and from enriched cardiomyocytes (Figure 1b). Recombination in all study animals was restricted to the heart and was not detected in tail DNA (Figure 1b). $C M$ GC-A KO and corresponding "flox/flox GC-A" littermates on a mixed C57BL6/129 background were studied. All experiments were approved by the local animal care committee and conform with NIH guidelines for the care and use of laboratory animals.

Isolation of cardiomyocytes for Southern blot and RT-PCR analysis and for measurement of single-cell contractility. Ventricular myocytes were isolated by collagenase digestion using an established protocol (20). After digestion, the ventricles were cut into several pieces and subjected to gentle agitation through a nylon mesh to separate the myocytes. For Southern blotting, isolated myocytes were plated onto laminin-coated dishes in M199 medium. After 4 hours, the dishes were gently washed with medium, and adherent cells (predominantly cardiomyocytes, as indicated by their characteristic rod shape) were lysed for DNA extraction.

Analysis of GC-A mRNA expression in isolated cardiomyocytes by RT-PCR. For RT-PCR, 20-30 freshly isolated, single cardiomyocytes were collected under microscopic magnification and transferred into total RNA-extraction buffer (RNeasy Mini Kit; QIAGEN GmbH, Hilden, Germany). After cDNA synthesis (Advantage RT-for-PCR kit; CLONTECH Laboratories GmbH, Heidelberg, Germany), the following oligonucleotide primers were used to amplify GC-A: sense primer 5'-CTCAACATCACAGTAAATCACC (located in exon 1), and antisense primer $3^{\prime}$ CCTGAAGGCACCTGTCTCG (located in exon 5). GC-A 
transcripts were normalized to GAPDH. Product amplification was 45 cycles for GC-A and 25 cycles for GAPDH. PCR products were separated on $1.5 \%$ agarose gels containing ethidium bromide, and ODs were quantified by densitometry (ImageQuant software; Amersham Biosciences GmbH, Freiburg, Germany).

Measurement of single-cell contractility. Contractile parameters of freshly isolated, electrically stimulated $(0.5 \mathrm{~Hz})$ adult ventricular cardiomyocytes were measured with a video-edge detection system. After 15 minutes of baseline recordings, the effects of ANP (Bachem Distribution Services, Weil am Rhein, Germany) or the $\beta_{1}$-adrenoreceptor agonist isoproterenol (Sigma-Aldrich Chemie $\mathrm{GmbH}$, Deisenhofen, Germany) were tested.

Guanylyl cyclase assay. ANP-dependent guanylyl cyclase activity in membranes prepared from whole hearts was determined as previously described (16). To initiate cyclase activity, $100 \mu \mathrm{g}$ membrane protein was incubated in assay buffer $(25 \mathrm{mM}$ HEPES, $4 \mathrm{mM} \mathrm{MgCl}, 1 \mathrm{mM}$ 3-isobutyl-1-methylxanthine, $2 \mathrm{mM}$ ATP, $2 \mathrm{mM}$ GTP, $30 \mathrm{mM}$ phosphocreatine, $400 \mu \mathrm{g} / \mathrm{ml}$ creatine phosphokinase [185 U/mg], and $0.5 \mathrm{mg} / \mathrm{ml} \mathrm{BSA}$ ) at $37^{\circ} \mathrm{C}$, with or without ANP. At 10 minutes of incubation, the reaction was stopped by addition of ice-cold $100 \%$ (vol/vol) ethanol (final concentration 70\%). After centrifugation $\left(3,000 \mathrm{~g}, 5\right.$ minutes, $\left.4^{\circ} \mathrm{C}\right)$, the supernatants were dried in a speed-vacuum concentrator, resuspended in sodium acetate buffer $(50 \mathrm{mM}, \mathrm{pH} 6.0)$, and acetylated, and the cGMP content was determined by RIA (16).

Blood pressure measurements in anesthetized mice and tissue harvesting. CM GC-A KO and floxed GC-A littermates ( $n=14$ per sex and genotype, 4-6 months old) were anesthetized with $2 \%$ isoflurane, and arterial blood pressure was measured via a carotid artery PE-50 catheter connected to a Statham pressure transducer (AD Instruments GmbH, Spechbach, Germany). Thereafter, blood was collected via the arterial catheter for determination of circulating ANP levels (see below), and the mice were sacrificed. The hearts were weighed, and the atria and right ventricular free wall were dissected away from the left ventricle inclusive of the septum. The individual chambers were weighed and either frozen in liquid nitrogen (for DNA or RNA extraction) or fixed in $4 \%$ buffered formaldehyde (for histology).

Histology and morphometrical analyses. Formaldehydefixed ventricles were embedded in paraffin, and sections were stained with $\mathrm{H} \& \mathrm{E}$, periodic acid Schiff (PAS; to discriminate cardiomyocyte cell borders), or $0.1 \%$ picrosirius red (for collagen). Photomicrographs of the sections were evaluated by a computer-assisted image analysis system (13), with the investigator blinded to the genotypes. The mean cardiomyocyte diameters were calculated by measurement of 150 cells per specimen (16 hearts per genotype) in the region of the cell nucleus. Interstitial collagen fractions were calculated as the ratio between the collagen area and the total ventricular area in the corresponding section, in percent (13).

Northern blot analysis. Ventricular mRNA levels of ANP, $\alpha$-skeletal actin ( $\alpha$-sk-actin), $\alpha M H C$, and $\beta$ MHC were estimated from $20 \mu \mathrm{g}$ total RNA (13). GAPDH was used for normalization. Specific mouse cDNA probes were labeled with $\left[\alpha^{-32} \mathrm{P}\right] \mathrm{dCTP}$; oligonucleotides for $\beta \mathrm{MHC}$ were labeled with $\left[\gamma^{32} \mathrm{P}\right] \mathrm{ATP}$. Radioactive signals were visualized in a PhosphorImager (Amersham Biosciences Europe $\mathrm{GmbH}$ ) and quantified by ImageQuant software (13).

Assay of plasma ANP levels. Plasma samples were extracted with Sep-Pak $\mathrm{C}_{18}$ cartridges (Waters GmbH, Eschborn, Germany; distributed by Immundiagnostik AG, Bensheim, Germany), and ANP contents were determined by a commercially available RIA (Peninsula Laboratories Inc., San Carlos, California, USA; distributed by Bachem Distribution Services).

Cardiac contractile responses to dobutamine in vivo. We observed in pilot experiments that, in our mouse strain under isoflurane anesthesia, dobutamine decreased
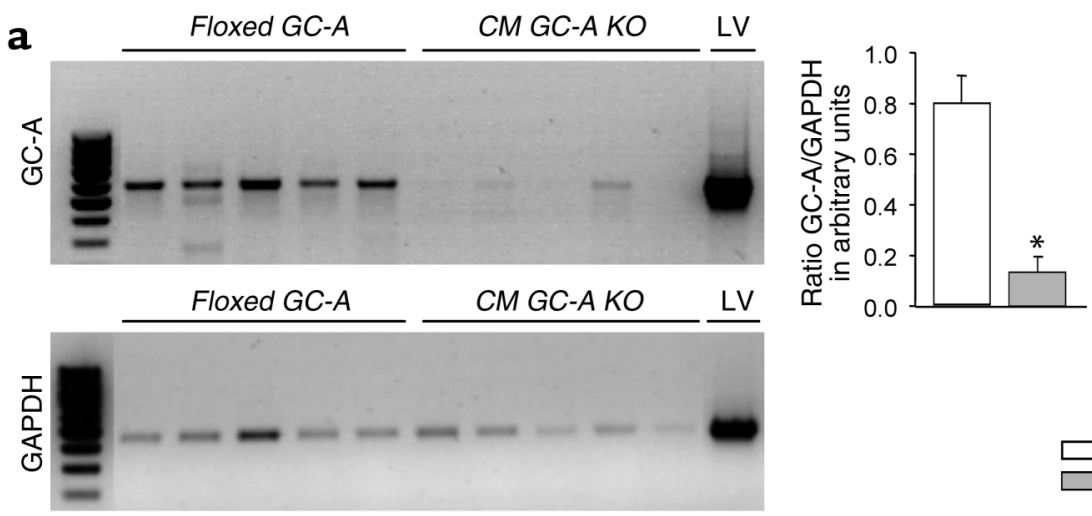

b

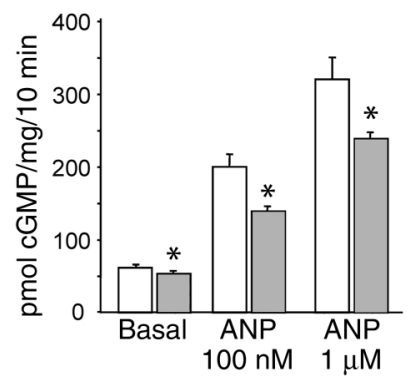

Floxed GC-A

CM GC-A KO

\section{Figure 2}

Demonstration of the GC-A deletion at the mRNA and protein level. (a) RT-PCR analyses of GC-A and GAPDH mRNA in preparations of isolated adult ventricular cardiomyocytes from floxed GC-A and CM GC-A KO mice (20-30 single cells were collected from each heart; five hearts were studied per genotype). Left: Ethidium bromide visualization of RT-PCR products. Left ventricle (LV) of a floxed GC-A mouse was used as positive control. Right: GC-A signal intensities were normalized to GAPDH. (b) Basal and ANP-stimulated guanylyl cyclase activity of plasma membranes isolated from whole hearts of floxed GC-A and CM GC-A KO mice. Enzymatic activity is expressed as picomoles of cGMP formed per milligram of protein over 10 minutes $(n=6)$. ${ }^{*} P<0.05$ vs. floxed GC- $A$. 


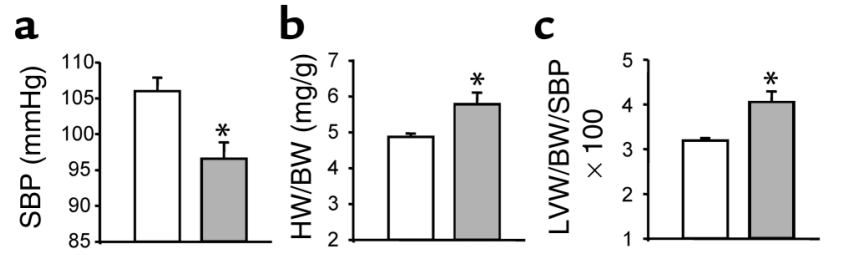

$\square$ Floxed GC-A

$\square C M G C-A K O$

\section{Figure 3}

Effect of cardiomyocyte-restricted disruption of the GC-A gene on systolic blood pressure (SBP) (a), heart weight/body weight $(\mathrm{HW} / \mathrm{BW})$ ratios $(\mathbf{b})$, and the relationship between left ventricular weight/body weight (LVW/BW) ratios and SBP $(\mathbf{c})(n=14$ per sex and genotype, $n=28$ per group, ${ }^{*} P<0.05$ vs. floxed GC-A).

blood pressure, confirming previous observations (21). Therefore, we evaluated the cardiac responses to dobutamine in mice anesthetized with tribromoethanol (14 $\mu \mathrm{l}$ of $2.5 \%$ solution per gram body weight, intraperitoneally; six mice per genotype, all 6-7 months old). Left ventricular catheterization was performed with a $1.4 \mathrm{~F}$ micromanometer-tipped catheter. Increasing doses of dobutamine were infused via the jugular vein, and hemodynamic measurements were performed for each dose after approximately 2 minutes.

Transverse aortic constriction. Mice (6-8 weeks old, 18-22 g) were anesthetized with a combination of ketamine (50 $\mathrm{mg} / \mathrm{kg}$ body weight, intraperitoneally) and isoflurane (2.5\%). Pressure overload was surgically induced by transverse aortic constriction (TAC) as previously described (22). Sham-operated animals underwent an identical surgical procedure except that ligation of the aorta was not performed. Ten days after TAC or the sham procedure, animals were anesthetized with $2 \%$ isoflurane. Pressure-transducing catheters were introduced into the right carotid and left femoral arteries, and systolic pressure before and after the stenosis was measured. To assess the effect on cardiac contractile function, in addi- tional experiments 10-14 days after TAC, left ventricular pressures were measured in isoflurane-anesthetized mice by in vivo catheterization.

Statistics. Results are expressed as the mean \pm SEM. Differences between CM GC-A KO and floxed GC-A mice were determined with an unpaired Student's $t$ test. $P$ values of less than 0.05 were considered statistically significant.

\section{Results}

Cardiomyocyte-selective deletion of the floxed GC-A gene. Disruption of the GC-A gene in the hearts of CM GC-A $K O$ mice was demonstrated by Southern blotting and RT-PCR. Cardiac DNA from flox/flox GC-A and CM GC-A KO mice was extracted, digested with BamHI, and hybridized to visualize the nonrecombined floxed allele and the recombined floxed-out allele. As shown in Figure 1, b and c, $23 \% \pm 4 \%$ of the alleles in cardiac samples from $C M$ GC-A KO mice have undergone recombination. Of note, entire cardiac tissue contains many other cells besides cardiomyocytes, such as vascular endothelial and smooth muscle cells and interstitial fibroblasts. All these cell types express GC-A (2, 4,8 ), but only the GC-A gene expressed in cardiomyocytes undergoes $\alpha \mathrm{MHC}$-Cre-mediated recombination. In fact, only $14 \%$ of mouse ventricular cells are cardiomyocytes, and $90-95 \%$ of these are binucleated (23), indicating that $24 \%$ of ventricular DNA originates from cardiomyocytes. The recombination of about $20 \%$ of the alleles in hearts from CM GC-A KO mice indicates that about $80 \%$ of the cardiomyocytes have undergone recombination. This deletion event was never demonstrated in genomic tail DNA obtained from the respective CM GC-A KO mice (Figure $1 \mathrm{~b}$ ). In enriched cardiomyocytes, the ratio of recombined to nonrecombined alleles was $55 \% \pm 3 \%$ (Figure 1, b and c). Since there is still substantial nonmyocyte contamination in this preparation, this number represents a lower estimate of the efficiency of

\section{Figure 4}

Histological analysis of floxed GC-A and CM GC-A KO hearts. Cardiomyocyte diameters and interstitial collagen fractions were estimated by quantitative morphometry of PAS-stained (a) or Sirius red-stained (b) ventricular sections, respectively. Black lines (a) indicate cardiomyocyte diameters in the region of the cell nucleus, which were significantly increased in CM GC-A KO hearts $(\mathbf{c})$, without changes in interstitial collagen (d) $\left(n=16,{ }^{*} P<0.05\right.$ vs. floxed GC-A). a
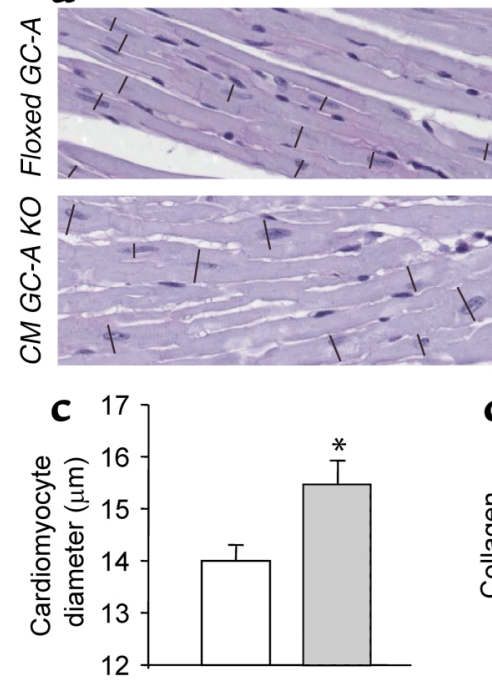

b
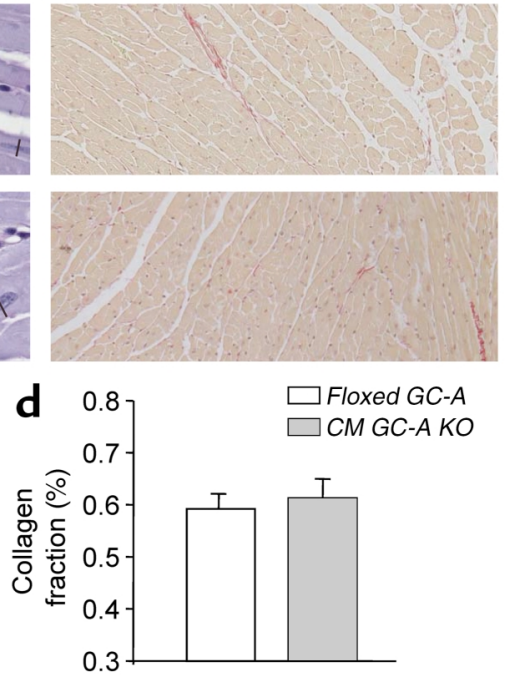


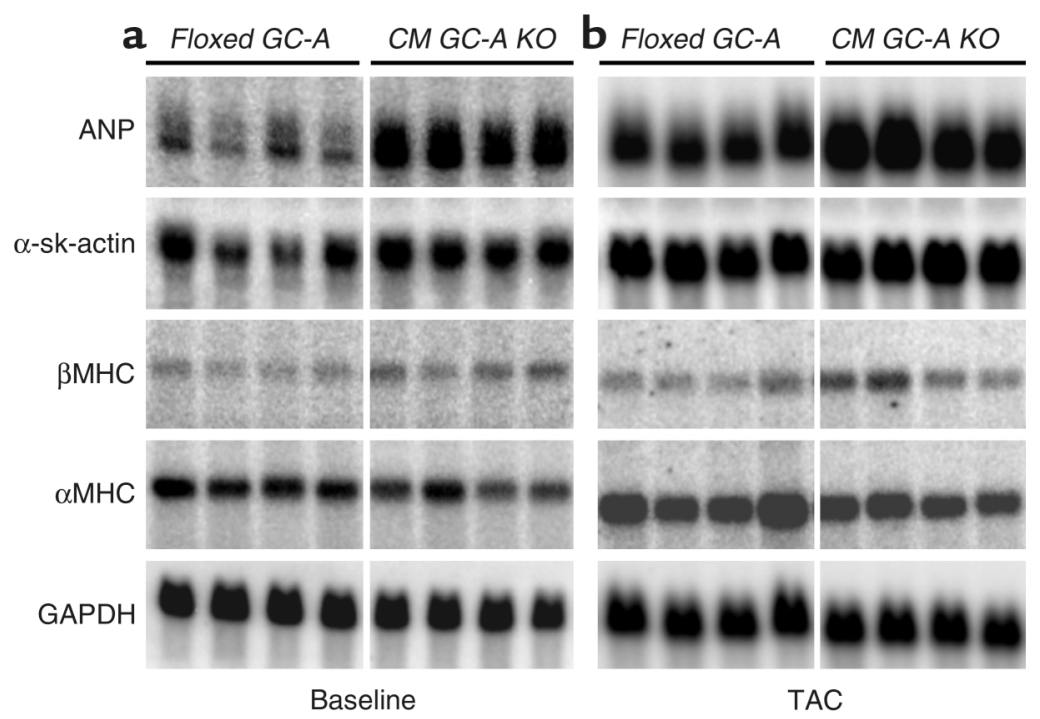

Figure 5

Representative Northern blots. Messenger RNA expression levels of ANP, $\alpha$-sk-actin, $\alpha M H C$, and $\beta M H C$, as well as GAPDH, in cardiac ventricles of floxed GC-A and CM GC-A KO mice under baseline conditions (a) and after TAC (b).

Cre-mediated recombination in the cardiomyocyte population. Indeed, semiquantitative RT-PCR demonstrated that the expression of GC-A mRNA in single cardiomyocytes of $C M G C-A K O$ mice is reduced by about $80 \%$ (Figure 2a). The CM GC-A KO mice grew to adulthood without signs of cardiac dysfunction and were grossly indistinguishable from their floxed GC-A littermates. Also, spontaneous lethality was not different between genotypes.

Decreased GC-A activity in cardiac membranes from $C M$ GC-A KO mice. To further demonstrate the deletion of GC-A, membrane-bound guanylyl cyclase activity was assayed in hearts from floxed GC-A and CM GC-A KO mice. Figure $2 \mathrm{~b}$ demonstrates that both basal and ANP-stimulated enzyme activities in CM GC- $A$ KO hearts were significantly reduced, by approximately $18 \%$ and $30 \%$, respectively. The high resting GC-A activity derives from vascular endothelial and smooth muscle cells as well as fibroblasts, which are all known to express GC-A and, as mentioned, constitute the predominant cell population in these whole-heart preparations.

CM GC-A KO mice exhibit decreased arterial blood pressure and cardiomyocyte bypertrophy. As shown in Figure 3a, systolic blood pressure of CM GC-A KO mice was slightly but significantly lower than that of age- and sex-matched floxed GC-A littermates, by about $7-10 \mathrm{mmHg}(P<0.01)$. In spite of this, there was a significant increase in the ratio of heart weight (HW) to body weight (BW) of CM GC-A $K O$ versus floxed $G C-A$ littermates $(P=0.012)$ (Figure $3 \mathrm{~b}$ ). As a result, the relationship between the left ventricular weight/body weight (LVW/BW) ratios and the systolic blood pressure levels differed significantly between genotypes (Figure 3c). Because the same relative changes were seen in both sexes, data from males and females were combined ( $n=14$ mice per sex and genotype).
Morphometrical analyses of PASstained sections demonstrated that cell diameters of ventricular cardiomyocytes from CM GC-A KO mice were significantly increased compared with those of their floxed GC-A littermates $(15.44 \pm 0.45 \mu \mathrm{m}$ and $14.04 \pm 0.3 \mu \mathrm{m}$, respectively; $P=0.018$ ) (Figure 4, a and c). Ventricular collagen fractions, quantitated on Sirius red-stained sections, were similar in both genotypes $(0.62 \% \pm 0.03 \%$ in CM GC-A KO and $0.59 \% \pm 0.02 \%$ in floxed GC-A mice) (Figure 4, b and d).

Increased expression of cardiac bypertrophy genes in CM GC-A KO mice. Next, we examined ventricular mRNA expression of hypertrophy markers. Northern blot analyses revealed that $C M$ GC- $A$ KO mice exhibit increased ventricular expression levels of ANP, $\alpha$-sk-actin, and $\beta$ MHC (increased $4.9 \pm 0.6$-fold, $1.7 \pm 0.2$-fold, and $2 \pm 0.6$-fold, respectively; $P<0.05$ vs. floxed $G C-A$ ) (Figure $5 a) . \alpha M H C$ levels were not changed (Figure 5a). The $\beta \mathrm{MHC} / \alpha \mathrm{MHC}$ ratio was increased $2.7 \pm 0.9$-fold $(n=8, P<0.05$ vs. floxed $G C-A)$.

CM GC-A KO mice exbibit increased circulating plasma ANP levels. To elucidate how the increased ventricular ANP mRNA levels in CM GC-A KO mice affect circulating concentrations, plasma levels were measured by RIA. As shown in Figure 6, plasma ANP levels were increased more than twofold in CM GC-A KO as compared with floxed GC-A littermates.

CM GC-A KO mice show preserved cardiac contractility but decreased cardiac relaxation. Heart rate and left ventricular contractility (Figure 7, a-c) were similar in CM GC-A $K O$ and floxed GC-A littermates ( $n=6$ per genotype, all 6-7 months old). Also, the chronotropic and inotropic responses to the $\beta$-agonist dobutamine were preserved (Figure 7, a-c). In contrast, the baseline maximal relaxation rates and the lusitropic responses of CM GC-A KO hearts to low levels of $\beta$-adrenergic stimulation were significantly decreased (Figure 7d).

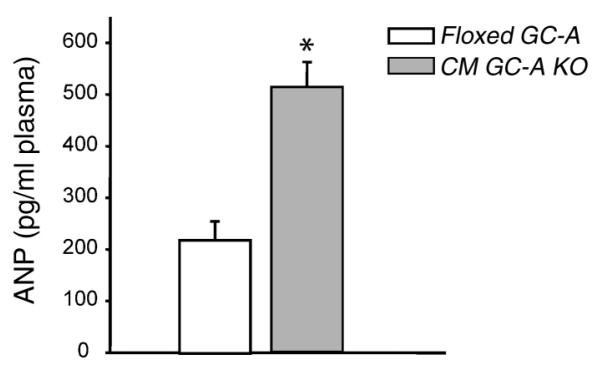

\section{Figure 6}

Plasma concentrations of ANP in CM GC-A KO mice compared with their floxed GC-A littermates $\left(n=18,{ }^{*} P<0.05\right.$ vs. floxed GC-A). 

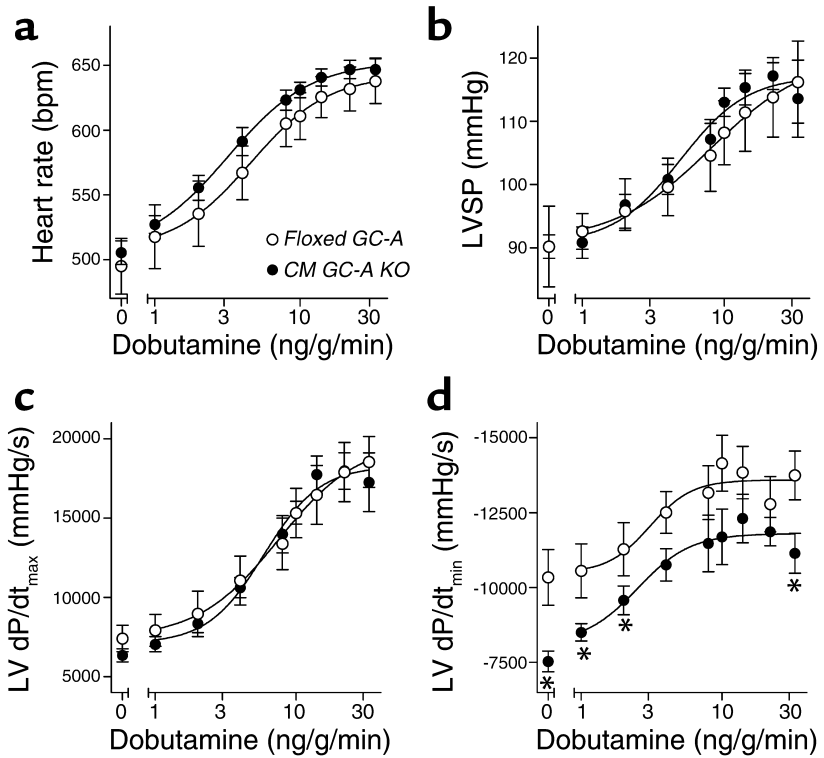

\section{Figure 7}

In vivo hemodynamic measurements of cardiac function by left ventricular catheterization. Heart rate (a), peak left ventricular systolic pressure (LVSP) (b), and the maximal rates of contraction $\left(\mathrm{dP} / \mathrm{dt}_{\max }\right)$ (c) and relaxation $\left(\mathrm{dP} / \mathrm{dt}_{\min }\right)(\mathbf{d})$ are shown at baseline $(0)$ and during infusion of dobutamine in CM GC-A KO and floxed GC-A mice $\left(n=6,{ }^{*} P<0.05\right.$ vs. floxed GC-A).

Isolated cardiomyocytes from CM GC-A KO mice exhibit normal contractility. Because ventricular performance in vivo is a function of not only intrinsic myocyte contractility, but also many systemic parameters, the influence of GC-A ablation on cardiomyocyte contractility was also examined in isolated, electrically paced ventricular myocytes. Corroborating the histological data, videotaped images of single cells showed that the resting length and width of GC-A KO cardiomyocytes were significantly increased (Table 1). Baseline myocyte shortening was similar in both genotypes. ANP inhibited the systolic shortening of floxed GC-A cardiomyocytes by about $25 \%$; in myocytes from $C M$ GC-A KO mice, the effect of ANP was abolished. Isoproterenol increased cell shortening 2.7 -fold in both genotypes (Table 1).

CM GC-A KO mice have an enhanced cardiac bypertrophic response to pressure overload. To investigate the local role of ANP under conditions of pressure overload, mice from both genotypes were subjected to TAC. After 10 days of banding, the systolic blood pressure (proximal to the constriction) was lower in CM GC-A KO than in floxed $G C-A$ mice (Figure $8 \mathrm{a}$ ). Also, the induced pressure gradient was lower in $C M$ GC- $A \mathrm{KO}$ mice $(72 \pm 3 \mathrm{mmHg}$ vs. $92 \pm 3.4 \mathrm{mmHg}, P=0.02)$. In spite of this, the average $\mathrm{HW} / \mathrm{BW}$ and $\mathrm{LVW} / \mathrm{BW}$ ratios were greater in banded $C M$ GC-A KO than in floxed GC-A mice (HW/BW, $8.85 \pm 0.14$ vs. $7.67 \pm 0.2, P=0.002$ ) (Figure $8 b$ ). Figure $8 \mathrm{c}$ shows the plotted LVW $\triangle B W$ ratios of individual animals as a function of the systolic pressure proximal to the aortic constriction. For any total pressure load, the CM GC-A KO

mice have an enhanced hypertrophic response (Figure 8d), suggesting that this enhancement is not simply a function of blood pressure. The mortality rates were similar in both genotypes.

Sirius red staining of left ventricular paraffin sections revealed that TAC caused a slight increase in interstitial collagen fractions - in floxed GC-A mice from $0.56 \pm 0.03$ (sham-operated) to $0.61 \pm 0.05$ (by $8 \%$ ), and in CM GC-A $K O$ mice from $0.57 \pm 0.04$ to $0.69 \pm 0.05$ (by $24 \%$, $P<0.05)$. Thus, cardiac fibrosis in response to TAC was slightly enhanced in CM GC-A KO mice.

Left ventricles from aortic-banded floxed GC- $A$ and CM GC-A KO mice were also analyzed for hypertrophic marker gene expression by Northern blotting. As compared with respective sham-operated mice, the increases in gene expression levels in floxed GC-A and CM GC-A KO mice were as follows: for ANP, 12.1- and 6.6-fold, respectively; for $\alpha$-sk-actin, 2.6and 1.9-fold, respectively; and for $\beta / \alpha M H C, 6.5$ - and 4.9-fold, respectively. As indicated in Figure 5b, the absolute ventricular mRNA levels of ANP and the ratios of $\beta \mathrm{MHC}$ to $\alpha \mathrm{MHC}$ were significantly greater in banded CM GC-A KO than in floxed GC-A mice: $1.66 \pm 0.1$-fold and $1.72 \pm 0.2$-fold, respectively $(n=10$, $P<0.05$ vs. floxed GC- $A$ ).

CM GC-A KO mice have markedly impaired cardiac function after pressure overload. Last, given the lower arterial blood pressure observed in CM GC-A KO mice with TAC, we performed an additional series of experiments, using left ventricular catheterization to measure cardiac function in mice banded for 10-14 days. CM GC-A KO mice showed decreased left ventricular contractility and relaxation as compared with floxed GC-A littermates, as evidenced by a reduction of the maximal rate of left ventricular pressure increase $\left(\mathrm{dP} / \mathrm{dt}_{\max }\right)$ and decrease $\left(\mathrm{dP} / \mathrm{dt}_{\min }\right)$, as well as by an increased left ventricular end-diastolic pressure (Table 2). In addition, the ratios of lung weight (LW) and right ventricular weight (RVW) to BW were greater in TAC-operated CM GC-A KO than in floxed GC- $A$ mice (LW/BW, $19.7 \pm 1.5$ vs. $13.8 \pm 1.6, P<0.05$; RVW/BW, $2.0 \pm 0.1$ vs. $1.6 \pm 0.1, P<0.05$ vs. floxed $G C-A ; n=6)$, indicating congestive heart failure without clinical signs of right ventricular failure.

\section{Table 1}

Contractile parameters of isolated adult ventricular cardiomyocytes

\begin{tabular}{lcc}
\hline & Floxed GC-A mice & CM GC-A KO mice \\
Cell length $(\mu \mathrm{m})$ & $110 \pm 2.15$ & $124 \pm 2.55^{\mathrm{B}}$ \\
Cell width $(\mu \mathrm{m})$ & $24.3 \pm 0.9$ & $29.89 \pm 1.17^{\mathrm{B}}$ \\
\% Shortening at baseline & $3.69 \pm 0.21$ & $3.23 \pm 0.16$ \\
$100 \mathrm{nM}$ ANP & $2.77 \pm 0.12^{\mathrm{A}}$ & $3.17 \pm 0.17$ \\
$100 \mathrm{nM}$ isoproterenol & $10.16 \pm 0.22^{\mathrm{A}}$ & $8.53 \pm 0.43^{\mathrm{A}}$
\end{tabular}

The contractile measurements were performed by edge detection. Myocyte dimensions measured from the videotaped images were compared with a calibration micrometer on the microscope stage. The maximal magnitude of contraction was normalized to resting cell length and expressed as percentage of shortening. $n=8$ cells, obtained from eight mice per genotype; ${ }^{A} P<0.05$ vs. baseline, ${ }^{\mathrm{B} P}<0.05$ vs. floxed GC- $A$. 

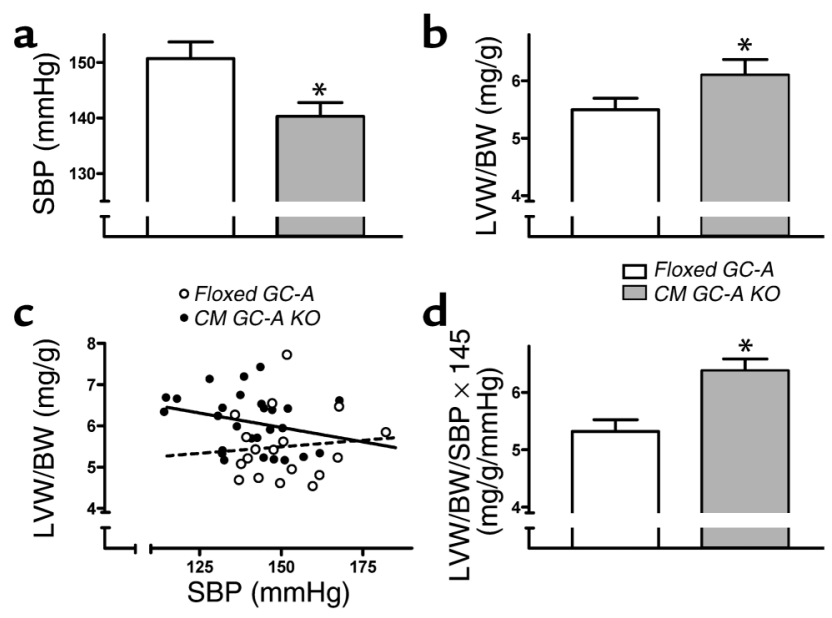

\section{Figure 8}

(a) TAC led to a substantial increase in systolic blood pressure (SBP) proximal to the stenosis. SBP was significantly lower in the CM GC-A $K O$ than in the floxed GC-A mice. For reference, pressures in shamoperated mice averaged $100 \pm 6 \mathrm{mmHg}$ (floxed GC-A) and $93 \pm 10$ $\mathrm{mmHg}(C M G C-A K O)$, indicating that a substantial pressure load was induced by TAC in both genotypes. (b) Despite the lower SBP, the CM GC-A KO mice had an increased LVW/BW index compared with the floxed GC-A mice. (c) The linear regression of SBP and LWW/BW index revealed that in the CM GC-A KO mice (filled circles and solid line), a stronger hypertrophic response in the heart correlated with lower blood pressures, while in the floxed GC-A mice (open circles and dashed line), blood pressure and LVW/BW index were positively correlated. (d) To compare the hypertrophic response independently of the differences in blood pressure in this figure, we divided the LVW/BW index of each animal by the individual SBP and normalized this value to the mean SBP of $145 \mathrm{mmHg}(n=18$ floxed GC- $A$ and 27 CM GC-A KO mice, ${ }^{*} P<0.05$ vs. floxed GC-A).

\section{Discussion}

The present study describes a cardiomyocyte-restricted inactivation of the GC-A gene (CM GC-A KO) using the loxP/Cre system. Chronic abolition of the local, cardiac effects of ANP in mice resulted in a slight but significant increase in cardiomyocyte size in the absence of interstitial fibrosis, accompanied by a marked increased in the expression of cardiac hypertrophy markers such as ANP, $\alpha$-sk-actin, and $\beta$ MHC. Cardiac contractility was preserved, but relaxation was slightly impaired. Surprisingly, CM GC-A KO mice exhibit a mild but significant arterial hypotension by $7-10$ $\mathrm{mmHg}$, which is probably related to the increased levels of ANP in the systemic circulation and the subsequently enhanced hypotensive effects of the peptide. Furthermore, CM GC-A KO mice have an increased hypertrophic response to aortic constriction-induced pressure load, accompanied by a marked deterioration of cardiac function. Taken together, these results suggest that ANP has a subtle but critical GC-A-mediated function to moderate the molecular program of hypertrophy in the heart.

Under physiological conditions, ANP is synthesized and secreted mainly by atrial myocytes (1). However, during cardiac hypertrophy, i.e., in response to arterial hypertension, ventricular myocytes undergo phenotypic modifications that result in the re-expression of fetal genes, in particular ANP and BNP (3). It was postulated that in this situation local ANP could be directly involved in the regulation of cardiomyocyte growth (8-13). To dissect the local actions of ANP in vivo, the GC-A gene was deleted specifically in cardiomyocytes. Floxed GC-A mice (16) were mated with mice expressing Cre recombinase in cardiomyocytes $\left(\alpha M H C-C r e^{t g}\right)(17)$. Based on Southern blot and RT-PCR analysis of whole hearts and purified cardiomyocytes obtained from the resulting CM GC-A KO offspring, we estimate the efficacy of the cardiomyocyte GC- $A$ gene deletion to be approximately $80 \%$. Furthermore, ANP-stimulated guanylyl cyclase activity was markedly reduced in CM GC-A KO hearts, by about $30 \%$; the high resting GC-A activity very likely derives from noncardiomyocyte cells in the heart. Indeed, the inhibitory effects of ANP on contractility of isolated cardiomyocytes in vitro are completely abolished in cardiac myocytes from CM GC-A KO mice, demonstrating the efficient deletion of GC-A.

Unexpectedly, loxP/Cre-mediated, cardiomyocyterestricted deletion of GC-A resulted in mild arterial hypotension, and yet cardiac weight and cardiomyocyte size (measured by histological and video-edge detection analyses) were significantly increased. Cardiomyocyte hypertrophy was accompanied by enhanced ventricular expression of the hypertrophy markers ANP, $\alpha$-skactin, and $\beta M H C$. In addition, CM GC-A KO mice have a greater cardiac hypertrophic response to TAC, which is accompanied by slightly enhanced cardiac fibrosis and marked impairment of cardiac function. These observations suggest that specific structural and/or molecular changes in GC-A-deficient cardiomyocytes compromise the adaptive responses to pressure overload. Taken together, our results suggest that ANP has a critical, GC-A-mediated local function to moderate cardiomyocyte growth under both normal and enhanced pressure-load conditions.

As demonstrated in vitro and in vivo, the resting contractile parameters of CM GC-A KO mice, and the inotropic responses of these mice to $\beta$-adrenergic stimulation, were preserved. Intriguingly, invasive hemodynamics revealed a slightly lower rate of cardiac relax-

\section{Table 2}

Cardiac function after TAC

$\begin{array}{lcc} & \text { Floxed GC-A mice } & \text { CM GC- } \text { A } \text { KO mice } \\ \text { Heart rate }(\text { beats/min }) & 541 \pm 14 & 550 \pm 10 \\ \mathrm{LVP}_{\max }(\mathrm{mmHg}) & 151 \pm 5 & 130 \pm 8 \\ \mathrm{LVEDP}(\mathrm{mmHg}) & 17 \pm 1.1 & 22.7 \pm 1.7^{\mathrm{A}} \\ \mathrm{LV} \mathrm{dP} / \mathrm{dt}_{\max }(\mathrm{mmHg} / \mathrm{s}) & 7,085 \pm 381 & 5,479 \pm 611^{\mathrm{A}} \\ \mathrm{LV} \mathrm{dP} / \mathrm{dt}_{\min }(\mathrm{mmHg} / \mathrm{s}) & -6,427 \pm 383 & -4,965 \pm 478^{\mathrm{A}}\end{array}$

Invasive hemodynamics in mice banded for 10-14 days shows that cardiac function in CM GC-A KO mice is markedly impaired as compared with that in floxed GC-A littermates. LVP $\mathrm{m}_{\max }$, left ventricular peak systolic pressure; LVEDP, left ventricular end-diastolic pressure; $\mathrm{LV} \mathrm{dP} / \mathrm{dt}_{\max }$ and $\mathrm{dP} / \mathrm{dt}_{\max }$, maximal rates of left ventricular pressure development and decline, respectively. $n=6$; ${ }^{A} P<0.05$ vs. floxed GC- $A$. 
ation both at baseline and under low levels of $\beta$-adrenergic stimulation. Interestingly, studies in humans (24) and rodents $(25,26)$ agree that ANP has little or no effect on cardiac contraction in vivo but directly accelerates relaxation. Deletion of cardiomyocyte GC-A may prevent this effect and thereby delay diastolic relaxation in CM GC-A KO mice. Obviously, impaired cardiac relaxation might also be related to specific structural and molecular changes within GC-A-deficient cardiomyocytes, an interesting question that we will address in future studies.

Since baseline cardiac contractility was normal and the mice clearly showed no clinical signs of cardiac dysfunction, we interpret the hypotensive effect of the cardiomyocyte-restricted ablation of GC-A as being caused by the markedly enhanced cardiac expression and secretion of ANP. Since the endocrine expression sites of GC-A (in the kidney, vasculature, adrenals, and CNS) are preserved, the increased circulating concentrations of ANP are likely to decrease blood pressure below normal. In this context the observation that CM GC-A KO mice exhibit increased cardiac expression and release of ANP is even more surprising, because the mild chronic arterial hypotension would be expected to induce, in turn, a homeostatic decrease in cardiac ANP secretion (3). In particular, two mechanisms might be responsible for this phenotype: (a) reactivation of a fetal gene expression program accompanying cardiomyocyte hypertrophy, and/or (b) abolition of an autoregulatory mechanism by which ANP inhibits its own secretion in an autocrine/paracrine GC-A/cGMP-mediated fashion (27). Indeed, cardiac overexpression of GC-A in mice drastically reduced ventricular ANP mRNA levels and concentrations (10). In the present study, the increases in ANP mRNA expression were disproportionally large compared with the increases in other hypertrophy marker genes, such as $\alpha$-sk-actin and $\beta$ MHC, suggesting that several mechanisms are involved.

Which cellular pathways are responsible for pressure-independent cardiac hypertrophy in CM GC-A KO mice? It is possible that high local levels of ANP and BNP could act via the other NP receptors expressed on cardiomyocytes, guanylyl cyclase-B (GC-B, a specific receptor for C-type natriuretic peptide) and NPR-C, to stimulate cardiomyocyte growth. However, the affinities of ANP and BNP are 100- to 1,000-fold lower for GC-B than for GC-A (28). Also, published studies in C-type natriuretic peptide (CNP) and NPR-C genedisrupted mice found no evidence of altered cardiomyocyte growth $(6,29)$. Alternatively, one could postulate that high local ANP and BNP concentrations enhanced the release of growth-stimulating factors from neighboring fibroblasts. However, both peptides inhibit the proliferative and synthetic activities of cardiac fibroblasts instead of accentuating them $(7,8)$. Hence, it is unlikely that the increased expression and release of ANP contributed to the enhanced cardiac hypertrophic responses. Instead, the most likely explanation for our observations is that the inhibition of direct, GC-A-mediated effects of ANP caused the hypertrophic phenotype.

The molecular mechanism(s) by which ANP and GC-A inhibit cardiac hypertrophy is not definitively known. ANP inhibits MAPK activation via cGMP (30). Additionally, a recent study showed that cGMP-dependent protein kinase I inhibits calcineurin/NFAT-mediated cardiomyocyte hypertrophy (31). However, these observations were in cultured neonatal cardiomyocytes, which share many but not all of the properties of adult cells. In our future studies we will take advantage of this newly developed genetic mouse model to further characterize the specific molecular pathways that mediate the inhibitory effects of ANP in adult cardiac myocytes.

In patients with heart hypertrophy and/or heart insufficiency, the plasma levels of ANP are increased, but the vasodilative and natriuretic responses to the peptide are markedly attenuated, suggesting impaired receptor or postreceptor responsiveness of GC-A (32). It is unknown whether these changes also affect GC-A in the heart. Our studies in CM GC-A KO mice indicate that an inhibition of the local cardiac ANP effects would accelerate the progression of cardiac hypertrophy and insufficiency, and they support further work to assess the importance of the ANP/GC-A system - particularly its local cardiac alterations - in human cardiovascular diseases.

\section{Acknowledgments}

The authors thank Bernd Zetsche, Melanie Voß, and Dorothe Möllmann for excellent technical assistance. This work was supported by the Bundesministerium für Bildung und Forschung (BMBF 01EC9801), the University of Münster (Interdisziplinäre Klinische Forschung, IZKF B12), and the Deutsche Forschungsgemeinschaft (SFB 556) (all to M. Kuhn). M. van Eickels received an institutional grant from the University of Bonn (Programm zur gezielten Forschungsförderung an der medizinischen Fakultät [BONFOR]).

1. de Bold, A.J., Borenstein, H.B., Veress, A.T., and Sonnenberg, H. 1981. A rapid and potent natriuretic response to intravenous injection of atrial myocardial extract in rats. Life Sci. 28:89-94.

2. Drewett, J.G., and Garbers, D.L. 1994. The family of guanylyl cyclase receptors and their ligands. Endocr. Rev. 13:135-162.

3. de Bold, A.J., et al. 2001. The physiological and pathophysiological modulation of the endocrine function of the heart. Can. J. Physiol. Pharmacol. 79:705-714.

4. Brenner, B.M., Ballermann, B.J., Gunning, M.E., and Zeidel, M.L. 1990 Diverse biological actions of atrial natriuretic peptide. Pharmacol. Rev. 70:665-699.

5. Murthy, K.S., et al. 2000. G(i-1)/G(i-2)-dependent signaling by singletransmembrane natriuretic peptide clearance receptor. Am. J. Physiol. 278:G974-G980.

6. Matsukawa, N., et al. 1999. The natriuretic peptide clearance receptor locally modulates the physiological effects of the natriuretic peptide system. Proc. Natl. Acad. Sci. U. S. A. 96:7403-7408.

7. Tamura, N., et al. 2000. Cardiac fibrosis in mice lacking brain natriuretic peptide. Proc. Natl. Acad. Sci. U. S. A. 97:4239-4244.

8. Calderone, A., Thaik, C.M., Takahashi, N., Chang, D.L.F., and Colucci, W.S. 1998. Nitric oxide, atrial natriuretic peptide, and cyclic GMP inhibit the growth-promoting effects of norepinephrine in cardiac myocytes and fibroblasts. J. Clin. Invest. 101:812-818.

9 . Horio, T., et al. 2000. Inhibitory regulation of hypertrophy by endogenous atrial natriuretic peptide in cultured cardiac myocytes. Hypertension. 35:19-24.

10. Kishimoto, I., Rossi, K., and Garbers, D.L. 2001. A genetic model provides evidence that the receptor for atrial natriuretic peptide (guanylyl 
cyclase-A) inhibits cardiac ventricular myocyte hypertrophy. Proc. Natl. Acad. Sci. U. S. A. 98:2703-2706.

11. Lopez, M.J., et al. 1995. Salt-resistant hypertension in mice lacking the guanylyl cyclase-A receptor for atrial natriuretic peptide. Nature. 378:65-68.

12. Knowles, J.W., et al. 2001. Pressure-independent enhancement of cardiac hypertrophy in natriuretic peptide receptor A-deficient mice. J. Clin. Invest. 107:975-984.

13. Kuhn, M., et al. 2002. Progressive cardiac hypertrophy and dysfunction in atrial natriuretic peptide receptor (GC-A) deficient mice. Heart. 87:368-374.

14. Shesely, E.G., et al. 1996. Elevated blood pressures in mice lacking endothelial nitric oxide synthase. Proc. Natl. Acad. Sci. U. S. A. 93:13176-13181.

15. John, S.W., et al. 1996. Blood pressure and fluid-electrolyte balance in mice with reduced or absent ANP. Am. J. Physiol. 271:R109-R114.

16. Holtwick, R., et al. 2002. Smooth muscle-selective deletion of guanylyl cyclase-A prevents the acute but not chronic effects of ANP on blood pressure. Proc. Natl. Acad. Sci. U. S. A. 99: 7142-7147.

17. Agah, R., et al. 1997. Gene recombination in postmitotic cells. Targeted expression of Cre recombinase provokes cardiac-restricted, site-specific rearrangement in adult ventricular muscle in vivo. J. Clin. Invest. 100:169-179.

18. Gutstein, D.E., et al. 2001. Conduction slowing and sudden arrythmic death in mice with cardiac-restricted inactivation of connexin43. Circ. Res. 88:333-339.

19. Gaussin, V., et al. 2002. Endocardial cushion and myocardial defects after cardiac myocyte-specific conditional deletion of the bone morphogenetic protein receptor ALK3. Proc. Natl. Acad. Sci. U. S. A. 99:2878-2883.

20. Kirchhefer, U., et al. 2001. Cardiac hypertrophy and impaired relaxation in transgenic mice overexpressing triadin 1. J. Biol. Chem. 276:4142-4149.

21. Kamphoven, J.H.J., et al. 2001. Cardiac remodeling and contractile func- tion in acid $\alpha$-glucosidase knockout mice. Physiol. Genomics. 5:171-179.

22. van Eickels, M., et al. 2001. 17 $\beta$-Estradiol attenuates the development of pressure-overload hypertrophy. Circulation. 104:1419-1423.

23. Soonpaa, M.H., Kim, K.K., Pajak, L., Franklin, M., and Field, L.J. 1996. Cardiomyocyte DNA synthesis and binucleation during murine development. Am. J. Physiol. 271:H2183-H2189.

24. Clarkson, P.B.M., Wheeldon, N.M., Macleod, C., Coutie, W., and MacDonald, T.M. 1995. Acute effects of atrial natriuretic peptide on left ventricular diastolic function. A pulse wave Doppler study in man. Eur. Heart J. 16:1710-1715.

25. Meulemans, A.L., Sipido, K.R., Sys, S.U., and Brutsaert, D.L. 1988. Atriopeptin III induces early relaxation of isolated mammalian papillary muscle. Circ. Res. 62:1171-1174.

26. Gyurko, R., Kuhlencordt, P., Fishman, M.C., and Huang, P.L. 2000. Modulation of mouse cardiac function in vivo by eNOS and ANP. Am. J. Physiol. 278:H971-H981.

27. Vesely, D.L., et al. 1994. Negative feedback of atrial natriuretic peptides. J. Clin. Endocrinol. Metab. 78:1128-1134.

28. Koller, K.J., and Goeddel, D.V. 1992. Molecular biology of the natriuretic peptides and their receptors. Circulation. 86:1081-1088.

29. Chusho, H., et al. 2001. Dwarfism and early death in mice lacking C-type natriuretic peptide. Proc. Natl. Acad. Sci. U. S. A. 98:4016-4021.

30. Sharma, G.D., et al. 2002. Expression of atrial natriuretic peptide receptor-A antagonizes the mitogen-activated protein kinases (Erk2 and P38 $\left.{ }^{\mathrm{MAPK}}\right)$ in cultured human vascular smooth muscle cells. Mol. Cell. Biochem. 233:165-173.

31. Fiedler, B., et al. 2002. Inhibition of calcineurin-NFAT hypertrophy signaling by cGMP-dependent protein kinase type I in cardiac myocytes. Proc. Natl. Acad. Sci. U. S. A. 99:11363-11368.

32. Hirooka, Y., et al. 1990. Attenuated forearm vasodilative response to intra-arterial atrial natriuretic peptide in patients with heart failure. Circulation. 82:147-153. 